\title{
Sinterização ultrarrápida por micro-ondas de compósitos particulados PZT/FCO preparados por mistura em ultrassom
}

\author{
(Ultra-fast microwave sintering of PZT/FCO particulate composites \\ prepared by ultrasonic mixing)
}

\author{
C. P. Fernandez ${ }^{1}$, F. L. Zabotto ${ }^{2}$, D. Garcia ${ }^{2}$, R. H. G. A. Kiminami ${ }^{1}$ \\ ${ }^{1} P P G-C E M$, Departamento de Engenharia de Materiais; ${ }^{2}$ Departamento de Física, \\ Universidade Federal de São Carlos, Rod. Washington Luiz, km 235, CP 676, São Carlos, SP 13565-905
}

\begin{abstract}
Resumo
Pós de $\mathrm{Pb}\left(\mathrm{Zr}_{0,53} \mathrm{Ti}_{0,47}\right) \mathrm{O}_{3}(\mathrm{PZT})$ e $\mathrm{Fe}_{2} \mathrm{CoO}_{4}(\mathrm{FCO})$, foram sintetizados separadamente pelo método Pechini e misturados em ultrassom, nas proporções molares 80/20 e 50/50 (PZT/FCO). Os compósitos preparados foram prensados e submetidos à sinterização em forno convencional e sinterização ultrarrápida assistida por micro-ondas. A caracterização estrutural e microestrutural das amostras foram realizadas por difração de raios X e microscopia eletrônica de varredura, respectivamente. Medidas de constante dielétrica em função da temperatura, resistividade elétrica e coeficiente de acoplamento magnetoelétrico foram também realizadas. Os resultados evidenciaram que o método de mistura do PZT com a FCO por ultrassom foi rápido e eficiente, gerando, após sinterização, compósitos particulados com conectividade global (0-3) e distribuição uniforme de grãos da fase ferromagnética (FCO) na matriz ferroelétrica (PZT). Da análise estrutural, verificou-se que a sinterização por micro-ondas propiciou um arranjo diferenciado no esquema de conectividade local na amostra (1-3) e (2-3), relacionada com a intensificação dos processos de difusão que ocorrem nesse tipo de sinterização principalmente em sistemas nanométricos. Pelos altos valores de resistividade obtidos, comprovou-se que a integridade das fases foi conservada nos dois métodos de sinterização, sendo mais eficiente na sinterização por micro-ondas, característica que garantiu o comportamento magnetoelétrico em todos os compósitos estudados neste trabalho. Os valores do campo $\mathrm{H}_{\max }$ foram dependentes da concentração da fase ferrita e da sinterização; para a composição 80/20 de 1,4 e 1,9 kOe, e para 50/50 de 3,5 e 3,0 kOe nas amostras sinterizadas por micro-ondas e convencionalmente, compatíveis com a literatura e que confirmaram a integridade das fases constituintes PZT e FCO.

Palavras-chave: PZT/FCO, compósito, mistura por ultrassom, micro-ondas.
\end{abstract}

\begin{abstract}
$\mathrm{Pb}\left(\mathrm{Zr}_{0.53} \mathrm{Ti}_{0.47}\right) \mathrm{O}_{3}(\mathrm{PZT})$ and $\mathrm{Fe}_{2} \mathrm{CoO}_{4}(\mathrm{FCO})$ powders were synthesized separately by the Pechini method and then ultrasonically mixed in molar proportions of 80/20 and 50/50 of PZT/FCO. The resulting composites were pressed and subjected to conventional and ultrafast microwave-assisted sintering. The structure and microstructure of the sintered samples were analyzed, respectively, by $X$-ray diffraction and scanning electron microscopy. The dielectric constant as a function of temperature, electrical resistivity and magnetoelectric coupling coefficient were measured. The results indicated that the ultrasonic mixing method applied to PZT and FCO was fast and efficient, and that sintering resulted in globally connected (0-3) particulate composites and uniform distribution of the ferromagnetic phase (FCO) grains in the ferroelectric matrix (PZT). The structural analysis indicated that microwave sintering changed the arrangement (1-3) of the material's local connectivity, which was attributed to the intensification of diffusion processes that occur in this type of sintering, particularly in nanometric systems. The high values of resistivity indicated that although both sintering methods preserved the integrity of the two phases, microwave sintering was more efficient, ensuring the magnetoelectric behavior of all the composites under study. The values of $H_{\text {max }}$ field were dependent on the ferrite phase concentration and sintering; 80/20 1.4 and $1.9 \mathrm{kOe}$, and 50/50 3.5 and $3.0 \mathrm{kOe}$ in the samples sintered by microwave and conventionally, consistent with the literature, which confirmed the integrity of the constituent phases PZT and FCO.
\end{abstract}

Keywords: PZT/FCO, composite, ultrasonic mixing, microwave.

\section{INTRODUÇÃO}

A partir do ano 2000, ressurge o interesse de uma nova classe de materiais, chamados multiferroicos magnetoelétricos, denominados assim por apresentar efeito de acoplamento magnetoelétrico (ME), tipicamente referido ao efeito magnetoelétrico linear manifestado como uma indução da magnetização por um campo elétrico ou polarização por um campo magnético [1,2], com extraordinário potencial para aplicações e otimização de 
dispositivos baseados nas propriedades ferroelétricas e ferromagnéticas, além do controle de tais propriedades pela aplicação de campos elétrico ou magnético [3]. Este tipo de efeito que apresentam os multiferroicos tem fascinado pesquisadores e engenheiros, não só desde uma perspectiva fundamental do conhecimento, mas sim desde o ponto de vista da aplicação. Imagine um mundo em que se pode controlar e manipular magnetismo com campos elétricos (os quais são intrinsecamente muito mais fáceis de usar em um dispositivo atual, especialmente em pequenas dimensões, e podem potencialmente fornecer rotas para menor potência/ consumo de energia nos sistemas), eliminando, assim, correntes e campos magnéticos [4].

Do ponto de vista de materiais constituintes, os materiais multiferroicos magnetoelétricos podem ser divididos em dois tipos: monofásicos e compósitos. Embora o efeito magnetoelétrico intrínseco exista em materiais monofásicos, a baixa temperatura crítica e/ou fraco acoplamento $\mathrm{ME}$ nesses materiais impedem suas aplicações práticas [5-8]. Alternativamente e com maior flexibilidade de preparação, compósitos multiferroicos ME feitos da combinação de fases ferroelétricas e ferromagnéticas têm atraído um significante interesse nos últimos anos, uma vez que nenhum dos materiais multiferroicos monofásicos existentes até hoje combina alta polarização elétrica e magnética, à temperatura ambiente. Em compósitos ME, nenhuma das fases constituintes apresenta o efeito ME, quando avaliada de forma individual, mas a interação cruzada entre elas pode produzir um notável efeito ME. O desenvolvimento de novos materiais e combinações de materiais é, portanto, um componente crítico que permite a exploração de tais fenômenos interessantes. Dentro desse contexto, os materiais multiferroicos, atualmente, propiciam desafios para o domínio de processos de síntese de novos materiais, implementação de diferentes métodos não convencionais de conformação, visando a otimização das propriedades derivadas do acoplamento dos sistemas ferroelétricos e ferromagnéticos e desenvolvimento de dispositivos. Contudo, em compósitos cerâmicos, o acoplamento magnetoelétrico é de natureza extrínseca, apresentando maior susceptibilidade a fatores como a síntese, método de mistura, sinterização, conectividade e propriedades das fases constituintes [9-11].

A maioria das publicações reportadas sobre compósitos cerâmicos ME utiliza a mistura mecânica com moinho de bolas, das fases ferroelétricas e ferromagnéticas obtidas separadamente, metodologia considerada como a origem e fonte comprometedora da obtenção do baixo efeito ME nos compósitos particulados, como consequência do deficiente controle dos defeitos mecânicos e dos baixos limites de início de difusão entre as fases [7]. Assim, o uso do ultrassom, utilizado como um método simples e alternativo de mistura, foi utilizado nesta pesquisa, visando evitar contaminação a nível atômico, maximizar a homogeneidade da mistura em menor tempo e garantir o acoplamento dos sistemas ferroelétricos e ferromagnéticos. Além disso, optou-se pela sinterização por micro-ondas do compósito magnetoelétrico PZT/FCO, visando evitar o crescimento de grãos durante a sinterização, dificilmente controlada pela sinterização convencional. Resultados da literatura demonstram que, pelo fato da sinterização ser realizada em apenas 10 a $20 \%$ do tempo gasto na sinterização convencional, e o aquecimento ser por micro-ondas, os mecanismos de sinterização diferem dos mecanismos da sinterização convencional, favorecendo inicialmente a densificação seguida do crescimento de grãos [12-15], o que permite o controle e a obtenção de microestruturas ultrafinas e uniformes em corpos cerâmicos. Assim, o objetivo deste trabalho foi avaliar o efeito da mistura por ultrassom de pós de $\mathrm{Pb}\left(\mathrm{Zr}_{0,53} \mathrm{Ti}_{0,47}\right) \mathrm{O}_{3}$ e $\mathrm{Fe}_{2} \mathrm{CoO}_{4}$ sintetizados pelo método Pechini e a sinterização por microondas, na microestrutura e propriedades magnetoelétricas dos compósitos magnetoelétricos particulados.

\section{MATERIAIS E MÉTODOS}

Os compósitos cerâmicos particulados PZT/FCO nas proporções molares 80/20 e 50/50 foram obtidos por mistura não convencional usando ultrassom, das fases sintetizadas, separadamente, mediante o método de precursor polimérico. $\mathrm{Na}$ síntese da $\mathrm{Fe}_{2} \mathrm{CoO}_{4}$ (FCO), inicialmente foi misturado etilenoglicol $\mathrm{C}_{2} \mathrm{H}_{6} \mathrm{O}_{2}$ (Merck, 99,5\%) com ácido cítrico $\mathrm{C}_{6} \mathrm{H}_{8} \mathrm{O}_{7}$ (Merck, 99,5\%) em uma relação molar 4:1. Simultaneamente, foram diluídos $\mathrm{Co}\left(\mathrm{NO}_{3}\right)_{2} \cdot 6 \mathrm{H}_{2} \mathrm{O}$ (Aldrich, $98 \%$ ) e $\mathrm{Fe}\left(\mathrm{NO}_{3}\right)_{3} \cdot 9 \mathrm{H}_{2} \mathrm{O}$ (Merck, 99,9\%) nas quantidades estequiométricas estabelecidas; as soluções foram adicionadas na solução de ácido cítrico e etilenoglicol e deixadas em agitação. A solução foi levada a uma condição básica pela adição de hidróxido de amônio $\left(\mathrm{NH}_{4} \mathrm{OH}\right)$, para favorecer a formação do citrato metálico. Na síntese de PZT, separadamente em álcool etílico, foram diluídos $\mathrm{C}_{16} \mathrm{H}_{36} \mathrm{O}_{4} \mathrm{Ti}$ (butóxido de titânio, Aldrich, 80\%, 1-butanol) e $\mathrm{C}_{16} \mathrm{H}_{36} \mathrm{O}_{4} \mathrm{Zr}$ (butóxido de zircônio, Aldrich, 97\%), mantidos em contínua agitação para evitar a precipitação dos butóxidos; as dissoluções de butóxido de titânio e butóxido de zircônio foram adicionadas na solução de ácido cítrico-etilenoglicol, previamente preparada, na mesma proporção molar usada na síntese da ferrita. Finalmente, o $\mathrm{Pb}\left(\mathrm{NO}_{3}\right)_{2}$ (Merck, 99,5\%) foi dissolvido em $0,5 \mathrm{M}$ de ácido nítrico e adicionado à solução contendo os cátions $\mathrm{Ti}^{4+}$ e $\mathrm{Zr}^{4+}$. Da mesma forma que no caso da ferrita, a solução de $\mathrm{Pb}-\mathrm{Zr}$-Ti foi levada a um pH básico.

As soluções obtidas tanto da ferrita como do PZT foram aquecidas a $120{ }^{\circ} \mathrm{C}$, para eliminar o solvente e promover a formação das resinas poliméricas; subsequentemente as resinas foram pré-calcinadas a $250^{\circ} \mathrm{C}$, durante $6 \mathrm{~h}$. O material sólido obtido de cada uma das fases foi desaglomerado e calcinado a $450{ }^{\circ} \mathrm{C}$ durante $2 \mathrm{~h}$ no caso da ferrita, e a $600{ }^{\circ} \mathrm{C}$, também durante 2 h, no caso do PZT. Após a confirmação da formação das fases individuais por difração de raios $\mathrm{X}$ e estabilização das características microestruturais de cada uma das fases usando área superficial e microscopia eletrônica de varredura, esses pós foram misturados nas proporções molares 80/20 e 50/50 (PZT/FCO), usandose um equipamento de ultrassom (Ultrasonic Processor, Sonics Vibra-cell, vc130, frequência de 120 kHz e 130 W de 
potência) durante 25 min em etanol como meio dispersante. Após a secagem realizada usando ar comprimido a fim de evitar aglomeração excessiva, os pós foram prensados em forma de discos cilíndricos com tamanho de $5 \mathrm{~mm}$ de diâmetro e $1 \mathrm{~mm}$ de espessura por prensagem isostática a frio (200 MPa). A medida por dilatometria da amostra prensada do compósito PZT/FCO foi realizada em um dilatômetro (Netzsch Tasc 414/2 Dilatometer 402 Ep) visando avaliar a retração linear relativa $(\Delta \mathrm{L} / \mathrm{Lo})$, à taxa constante de aquecimento de $10^{\circ} \mathrm{C} / \mathrm{min}$ até $1150^{\circ} \mathrm{C}$. A densidade relativa $\left(\mathrm{Q}_{\mathrm{i}}\right)$ durante o ensaio de dilatometria foi estimada com base na densidade a verde $\left(\varrho_{\text {verde }}\right)$ e utilizando a equação:

$$
\rho_{\mathrm{i}}=\frac{\rho_{\text {verde }}}{(1+\Delta \mathrm{L} / \mathrm{Lo})^{3}}
$$

As amostras prensadas foram submetidas à sinterização ultrarrápida assistida por micro-ondas e sinterização convencional. A sinterização por micro-ondas (SM) foi realizada em uma cavidade multimodal a $2,45 \mathrm{GHz}$ com magnéton de $6 \mathrm{~kW}$ (Cober Electronics, MS6K), com taxa de aquecimento de $100{ }^{\circ} \mathrm{C} / \mathrm{min}$ durante $15 \mathrm{~min}$ (em atmosfera de ar convencional), em intervalo de 1000 e $1150{ }^{\circ} \mathrm{C}$ e com tempo de duração do ciclo de sinterização de $\sim 50 \mathrm{~min}$ (aquecimento-resfriamento), tal como esquematizado na Fig. 1. Para comparação, as mesmas composições foram sinterizadas convencionalmente (SC) no intervalo de 1050 a $1200^{\circ} \mathrm{C}$ durante $3 \mathrm{~h}$, com taxa de aquecimento de $5^{\circ} \mathrm{C} / \mathrm{min}$ e tempo de duração do ciclo de sinterização de $\sim 12 \mathrm{~h}$, usandose para isso um forno tipo mufla (EDG 3600). Nesse caso, as amostras foram cobertas com um cadinho de $\mathrm{Al}_{2} \mathrm{O}_{3}$-mullita selado com pó de $\mathrm{PbZrO}_{3}+10 \% \mathrm{ZrO}_{2}$, para minimizar a volatilização de $\mathrm{PbO}$.

As análises de difração de raios X (DRX) e microscopia eletrônica de varredura (MEV) das amostras, tanto não sintetizadas como sinterizadas, foram realizadas utilizandose um difratômetro Rigaku D/II-B X-ray, com radiação de $\mathrm{CuK} \alpha$ como fonte, e um SEM-FEG (Phillips, XL30 FEG),

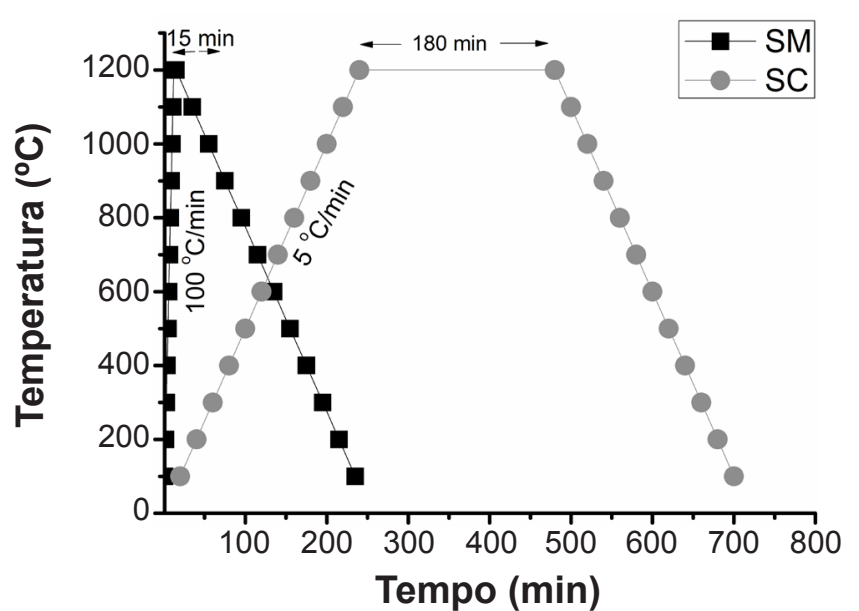

Figura 1: Curvas de queima por micro-ondas (SM) e sinterização convencional (SC).

[Figure 1: Firing curves for microwave (MS) and conventional (CS) sintering.] respectivamente. As medidas de adsorção de $\mathrm{N}_{2}$ (BET) para análise de área superficial $\left(\mathrm{S}_{\mathrm{BET}}\right)$ foram feitas com um BET Micrometrics, Gemini-2370, e o tamanho médio de partícula esférica, $D_{\mathrm{BET}}$, foi estimado pela relação $\mathrm{D}_{\mathrm{BET}}=6 / \mathrm{Q}_{\mathrm{th}} \cdot \mathrm{S}_{\mathrm{BET}}$, onde $\mathrm{Q}_{\text {th }}$ é a densidade teórica de cada uma das fases, 5,304 $\mathrm{g} / \mathrm{cm}^{3}$ para a ferrita e $8,006 \mathrm{~g} / \mathrm{cm}^{3}$ para PZT, de acordo com os arquivos JCPDS 33-0784 e 22-1086, respectivamente. A distribuição de tamanho de grãos foi analisada em imagens de MEV pelo método planimétrico, a partir do cálculo do número de grãos por unidade de área utilizando o programa Image Pro Plus.

A caracterização dielétrica das amostras sinterizadas foi realizada usando-se um analisador de impedância (HP 4194A) em função da temperatura; as amostras tiveram as superfícies polidas e eletrodos de ouro via sputtering foram depositados. As medidas foram realizadas na faixa de $50 \mathrm{e}$ $450{ }^{\circ} \mathrm{C}$ com taxa de aquecimento constante de $2{ }^{\circ} \mathrm{C} / \mathrm{min}$. As medidas de resistividade elétrica à temperatura ambiente, com campo aplicado dc, foram realizadas usando-se um eletrômetro Keithley 617. Finalmente, as amostras foram polarizadas eletricamente, à temperatura ambiente, com um campo elétrico de $2 \mathrm{kV} / \mathrm{cm}$ por 20 min e os coeficientes magnetoeléctricos, $\alpha_{33} \mathrm{ME}$, foram determinados a partir da medida do campo magnético, induzindo uma tensão através da amostra, usando-se um amplificador lock-in [16]. Nesse caso, foi aplicado um campo magnético dc variando entre -10 e +10 kOe, sobreposto a um campo magnético ac (5 Oe a $1 \mathrm{kHz}$ ).

\section{RESULTADOS E DISCUSSÃO}

A Fig. 2a apresenta os resultados de microscopia eletrônica de varredura após mistura por ultrassom durante 25 min dos pós de PZT e FCO na proporção 80/20, obtidos pelo método Pechini. Pôde-se notar a presença de aglomerados moles assimétricos com tamanhos maiores que $1 \mu \mathrm{m}$, característica típica dos pós obtidos pelo método Pechini, e partículas primárias entre 20 e 50 $\mathrm{nm}$ com morfologia esférica. Embora, na mistura PZT e FCO, tenha sido observada a presença de aglomerados, estes se apresentaram em menor tamanho dos que cada uma das fases (PZT e FCO) apresentou, tal como pode ser observado nas micrografias da Fig. 2b (inset), indicando que o tempo de mistura de 25 min utilizado favoreceu, além da mistura prevista, a ocorrência de desaglomeracão dos pós, permitindo assim uma mistura homogênea de ambas as fases. Assim, após o tempo de mistura de $25 \mathrm{~min}$ por ultrassom, sem a preocupação de contaminação, o tamanho dos aglomerados diminuiu em comparação com os pós de partida. Os resultados de difratometria de raios $\mathrm{X}$ de cada uma das fases constituintes sintetizadas mediante o método Pechini estão apresentados na Fig. 2b. Esses resultados confirmaram, em ambos os casos, a completa cristalização das fases obtidas: a fase $\mathrm{FCO}$ após calcinação a $450{ }^{\circ} \mathrm{C} / 2$ $\mathrm{h}$ e a fase PZT a $600{ }^{\circ} \mathrm{C} / 2 \mathrm{~h}$. Ambas as fases sintetizadas não apresentaram traços de fases secundárias e foram identificadas como fases principais $\mathrm{Fe}_{2} \mathrm{CoO}_{4}$ tipo espinélio, 

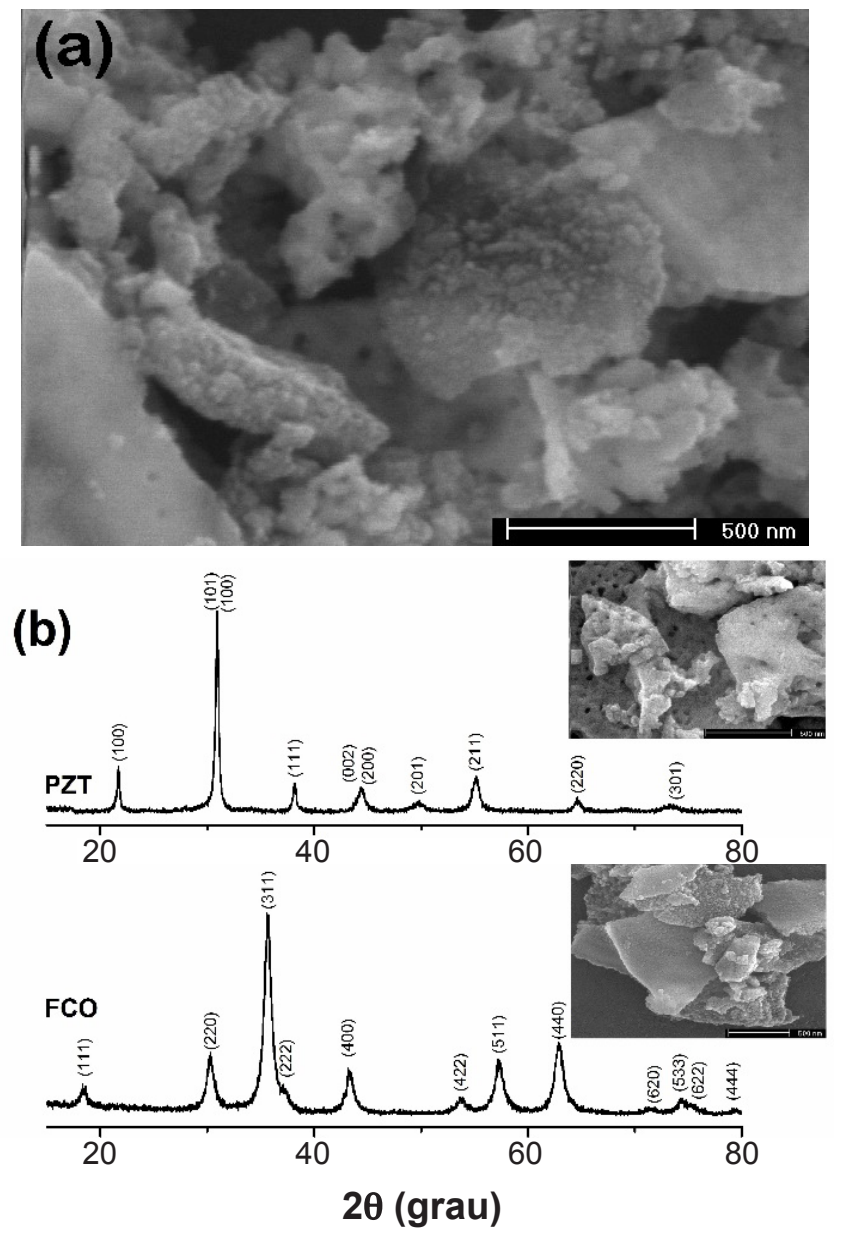

Figura 2: (a) Micrografia obtida por microscopia eletrônica de varredura da mistura por ultrassom dos pós de PZT e FCO, na proporção 80/20; (b) difratogramas de raios X de cada um dos pós constituintes, PZT e FCO, após a etapa de calcinação.

[Figure 2: (a) SEM micrograph of PZT and FCO powders ultrasonically mixed in the proportion of 80/20; (b) X-ray diffraction patterns of each constituent powder, PZT and FCO, after calcination.]

com simetria cúbica (JCPDS 22-1086), e $\mathrm{Pb}\left(\mathrm{Zr}_{0,53} \mathrm{Ti}_{0,47}\right) \mathrm{O}_{3}$ tipo perovskita (JCPDS 33-0784), com simetria tetragonal.

A partir dos resultados de DRX da Fig. 2b de ambos os pós, os tamanhos de cristalito foram calculados utilizando-se a fórmula de Scherrer, mediante a deconvolução gaussiana dos picos $\left(\mathrm{d}_{100}\right)$ e $\left(\mathrm{d}_{311}\right)$, respectivamente, de cada pó. Os resultados, após esse cálculo, foram de aproximadamente 41 e $18 \mathrm{~nm}$ para as fases PZT e FCO, respectivamente, conforme apresentado na Tabela I. Resultados similares foram obtidos após mistura por ultrassom durante 25 min do PZT e FCO na proporção 50/50, como era esperado. A Tabela I apresenta também os resultados da área específica superficial $\left(\mathrm{S}_{\mathrm{BET}}\right)$ e tamanho aproximado das partículas de ambas as fases, PZT e FCO. O PZT apresentou um valor de $5,2 \mathrm{~m}^{2} / \mathrm{g}$ de área superficial específica e tamanho de partículas próximo a 142 $\mathrm{nm}$, enquanto a $\mathrm{FCO}$ apresentou valores de $32,8 \mathrm{~m}^{2} / \mathrm{g}$ e 34 $\mathrm{nm}$, respectivamente. Comparando-se esses valores com o tamanho de cristalito calculado, 41 e $18 \mathrm{~nm}$ para as fases PZT e FCO, respectivamente, o resultado do tamanho das partículas correspondeu aproximadamente a duas ou três partículas aglomeradas ou partículas secundárias. A área superficial da mistura (Tabela I) foi de $5,2 \mathrm{~m}^{2} / \mathrm{g}$ para a composição 80/20, valor que coincide com o valor de área superficial da matriz (PZT). No caso da composição 50/50, observou-se que o valor da área superficial da mistura foi de $20,4 \mathrm{~m}^{2} / \mathrm{g}$, maior do que o da mistura $80 / 20$ como era de se esperar, devido ao aumento da proporção da fase FCO na composição.

Tabela I - Valores de área superficial, tamanho médio de partículas e tamanho de cristalito dos pós sintetizados pelo método Pechini e misturas $(80 / 20,50 / 50)$ preparadas em ultrassom.

[Table I - Surface area, particle and crystallite sizes of powders obtained by Pechini method, and mixtures (80/20, 50/50) prepared by ultrasonic mixing.]

\begin{tabular}{lccc}
\hline & $\begin{array}{c}\text { Área } \\
\text { superficial } \\
\text { BET }\left(\mathrm{m}^{2} / \mathrm{g}\right)\end{array}$ & $\begin{array}{c}\text { Tamanho médio } \\
\text { de partículas } \\
(\mathrm{nm})\end{array}$ & $\begin{array}{c}\text { Tamanho } \\
\text { de cristalito } \\
(\mathrm{nm})\end{array}$ \\
\hline PZT & 5,2 & 142 & 41 \\
$\mathrm{FCO}$ & 32,8 & 34 & 18 \\
$80 / 20$ & 5,2 & - & - \\
$50 / 50$ & 20,4 & - & - \\
\hline
\end{tabular}

A Fig. 3 apresenta a curva de retração linear em função de temperatura obtida por dilatometria com aquecimento convencional para a mistura PZT/FCO 80/20 prensada isostaticamente. Pôde-se observar que em $850{ }^{\circ} \mathrm{C}$ se deu o início do processo de retração, atingindo em $1050{ }^{\circ} \mathrm{C}$ uma retração da amostra de $10 \%$, associada ao início da densificação do compacto e à redução dos poros. Observouse que até $1125^{\circ} \mathrm{C}$, etapa intermediária, a retração foi de aproximadamente $18 \%$ e, no estágio final da sinterização, observou-se baixa taxa de retração. A retração total até $1150{ }^{\circ} \mathrm{C}$ foi em torno de $20 \%$, sendo que, além dos mecanismos difusionais de sinterização no compósito, pode ter ocorrido formação de uma pequena quantidade de fase líquida devido à fusão do $\mathrm{PbO}$, característica típica desse material, conforme também relatado em [17], no estudo de sinterização de compósitos particulados magnetoelétricos de $\mathrm{PZT} / \mathrm{Fe}_{2} \mathrm{NiO}_{4}$. A curva de densidade relativa em função da temperatura foi estimada considerando-se a retração linear, densidade teórica de $7,4 \mathrm{~g} / \mathrm{cm}^{3}$ e densidade a verde do material de $3,6 \mathrm{~g} / \mathrm{cm}^{3}(49,1 \%)$, resultando numa densidade de $90 \%$ da densidade teórica.

A partir desses resultados, as amostras foram sinterizadas em forno convencional e por micro-ondas em temperaturas entre 1000 e $1150{ }^{\circ} \mathrm{C}$. A Fig. 4 apresenta os resultados das curvas de densificação em função da temperatura, da composição 80/20 sinterizada tanto em forno convencional como por micro-ondas. Observou-se o aumento progressivo da densidade com o aumento da temperatura, atingindo valores de densidade relativa máxima de $\sim 94 \%$ e $91 \%$ nas amostras sinterizadas em forno convencional e por micro- 


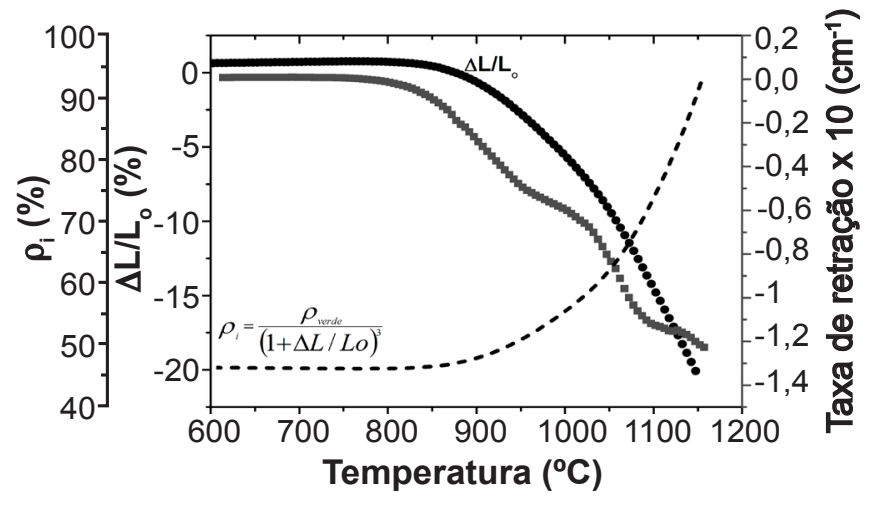

Figura 3: Retração linear relativa $\left(\Delta \mathrm{L} / \mathrm{L}_{\mathrm{o}}\right)$, taxa de retração e densidade relativa $\left(\varrho_{\mathrm{i}}\right)$ em função da temperatura do compósito 80/20, obtida por dilatometria com aquecimento convencional.

[Figure 3: Dilatometric measurement of relative linear retraction $\left(\Delta L / L_{o}\right)$, retraction rate, and relative density $\left(\varrho_{i}\right)$ vs. temperature of the 80/20 composite sintered by the conventional route.]

ondas, respectivamente. Acima de $1125{ }^{\circ} \mathrm{C}$, temperatura que correspondeu à temperatura do início do estágio final de sinterização, segundo a curva de dilatometria da Fig. 3 , observou-se na sinterização convencional uma queda abrupta na densidade, atribuída à volatilização e perda de $\mathrm{PbO}$ na fase PZT; já na sinterização por micro-ondas, o valor da densidade relativa nessas temperaturas permaneceu aproximadamente constante até $1200^{\circ} \mathrm{C}$, indicando mínima perda de $\mathrm{PbO}$ em altas temperaturas sob o ultrarrápido aquecimento assistido por micro-ondas. Esse resultado abre um excelente potencial da queima assistida por microondas de ferroelétricos à base de chumbo, dispensando o controle de atmosfera saturada de $\mathrm{PbO}$, e, principalmente, da sinterização ocorrer em um menor período de tempo. As micrografias de MEV apresentadas na Fig. 4 correspondem aos compósitos PZT/FCO (80/20) sinterizados a 1100 e $1125{ }^{\circ} \mathrm{C}$ tanto em forno convencional $(\mathrm{a}, \mathrm{b})$ como por micro-ondas (c,d). Pôde-se observar que, com o aumento da temperatura de sinterização, a percolação da fase ferrita foi mais evidente nos compósitos sinterizados por microondas. Esse efeito pode ser atribuído ao aquecimento ser ultrarrápido com forte evidência dos mecanismos de densificação acentuados, $1100{ }^{\circ} \mathrm{C} / 15 \mathrm{~min}$ a $1125^{\circ} \mathrm{C} / 15 \mathrm{~min}$, passando a densificação das amostras de $\sim 70 \%$ para $91 \%$ de densidade relativa. Esses resultados foram considerados excelentes, considerando-se a diferença dos coeficientes de expansão térmica de cada uma das fases constituintes, que dificulta o processo de densificação de sistemas bifásicos $\left(8 \times 10^{-6}{ }^{\circ} \mathrm{C}^{-1}\right.$ para a FCO $[18]$ e $1,2 \times 10^{-6}{ }^{\circ} \mathrm{C}^{-1}$ para a fase PZT [19]).

A Fig. 5 apresenta os resultados de difratometria de raios X dos compósitos PZT/FCO (80/20 e 50/50), sinterizados por micro-ondas e convencionalmente. Todas as linhas de difração corresponderam às fases cristalinas de $\mathrm{Fe}_{2} \mathrm{CoO}_{4}$ (JCPDS 22-1086) e $\mathrm{Pb}\left(\mathrm{Zr}_{0,47} \mathrm{Ti}_{0,53}\right) \mathrm{O}_{3}$ (JCPDS 33-0784) sem presença de fases secundárias, que eventualmente poderiam ocorrer decorrente da interdifusão das fases PZT e FCO. No caso dos compósitos 80/20 (Fig. 5a) e 50/50 (Fig. 5b) sinterizados por micro-ondas, foi observada presença de alguns traços da fase $\mathrm{ZrO}_{2}$ (JCPDS 37-1484) pela pequena perda de $\mathrm{PbO}$ em ambos os sistemas, devido a que, neste caso, foi dispensado o sistema de atmosfera saturada de $\mathrm{PbO}$.

Os resultados da análise microestrutural, a partir de microscopia eletrônica de varredura, dos compósitos $80 / 20$ e $50 / 50$ sinterizados a $1125{ }^{\circ} \mathrm{C}$, tanto por micro-

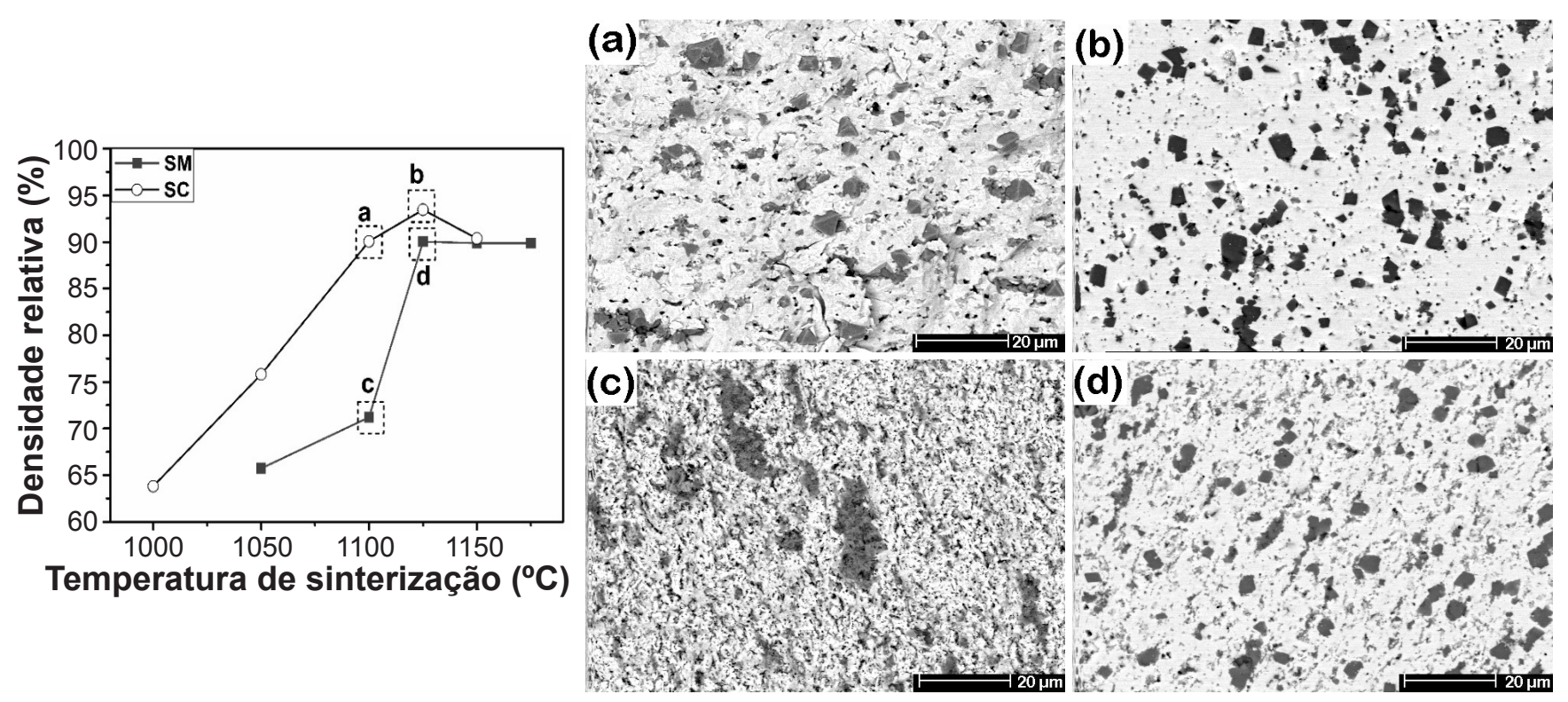

Figura 4: Esquerda: curvas de densidade relativa em função da temperatura do compósito PZT/FCO (80/20) sinterizado convencionalmente (SC) e por micro-ondas (SM). Direita: micrografias de MEV dos pontos marcados sobre as curvas de densidade $(\mathrm{a}, \mathrm{b}) \mathrm{SC}$ e (c,d) SM.

[Figure 4: Left: relative density curves as a function of temperature of PZT/FCO (80/20) composite, sintered conventionally (SC) and by microwave (SM). Right: SEM micrographs of the marked points in the density curves $S C(a, b)$ and $S M(c, d)$.] 

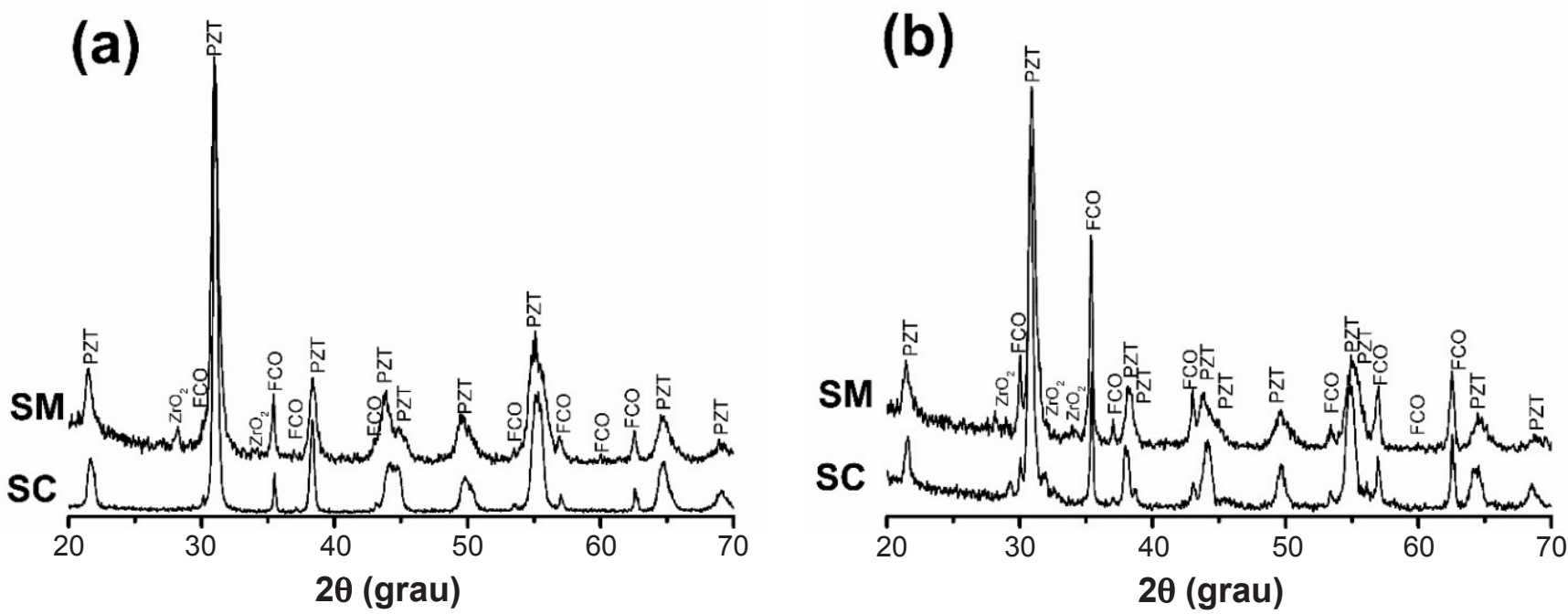

Figura 5: Difratogramas de raios X do compósito PZT/FCO (a) 80/20 e (b) 50/50, sinterizado por micro-ondas (SM) e forno convencional (SC). [Figure 5: X-ray diffraction patterns of PZT/FCO composite (a) 80/20 and (b) 50/50, sintered by the microwave (MS) and conventional (CS) routes.]
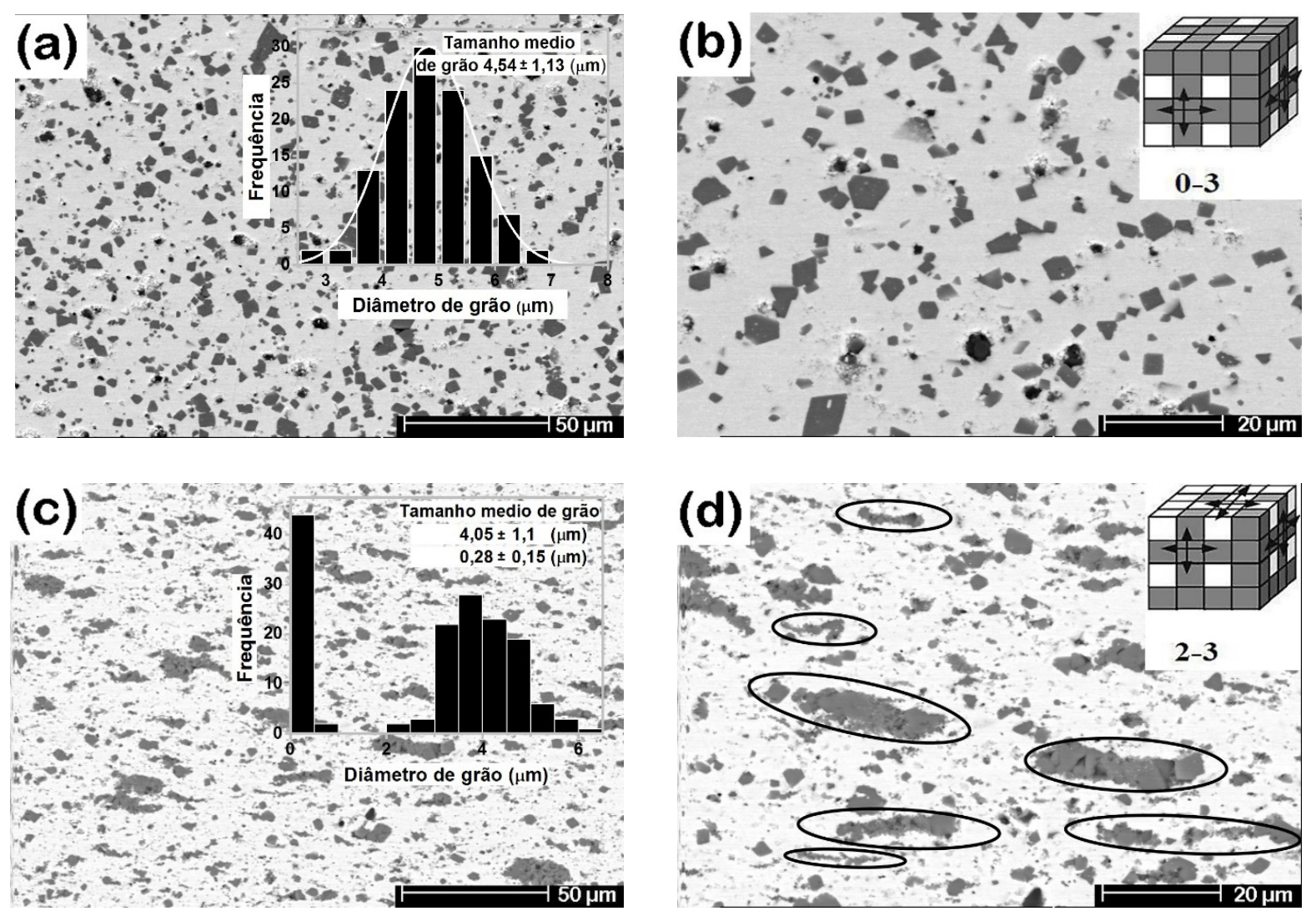

Figura 6: Micrografias obtidas por microscopia eletrônica de varredura da superfície polida do compósito PZT/FCO (80/20) sinterizado $(\mathrm{a}, \mathrm{b})$ em forno convencional e $(\mathrm{c}, \mathrm{d})$ por micro-ondas a $1125^{\circ} \mathrm{C}$ (região clara: $\mathrm{PZT}$, região escura: $\left.\mathrm{FCO}\right)$; inset $(\mathrm{a}, \mathrm{c})$ : distribuição de tamanho de grão da fase FCO.

[Figure 6: SEM micrographs of the polished surface of PZT/FCO (80/20) composite sintered (a,b) in a conventional furnace, and $(c, d)$ by microwave at $1125^{\circ} \mathrm{C}$ (bright grains: PZT, dark grains: $\left.F C O\right)$; inset (a,c): grain size distribution of FCO phase.]

ondas como convencionalmente, estão apresentados nas Figs. 6 e 7, respectivamente. Nas amostras sinterizadas convencionalmente, Figs. 6a,b e 7a,b, observou-se uma distribuição uniforme da fase ferrita na matriz de PZT, com um adequado controle da percolação da fase ferrita (razoável separação entre os grãos da fase) principalmente 

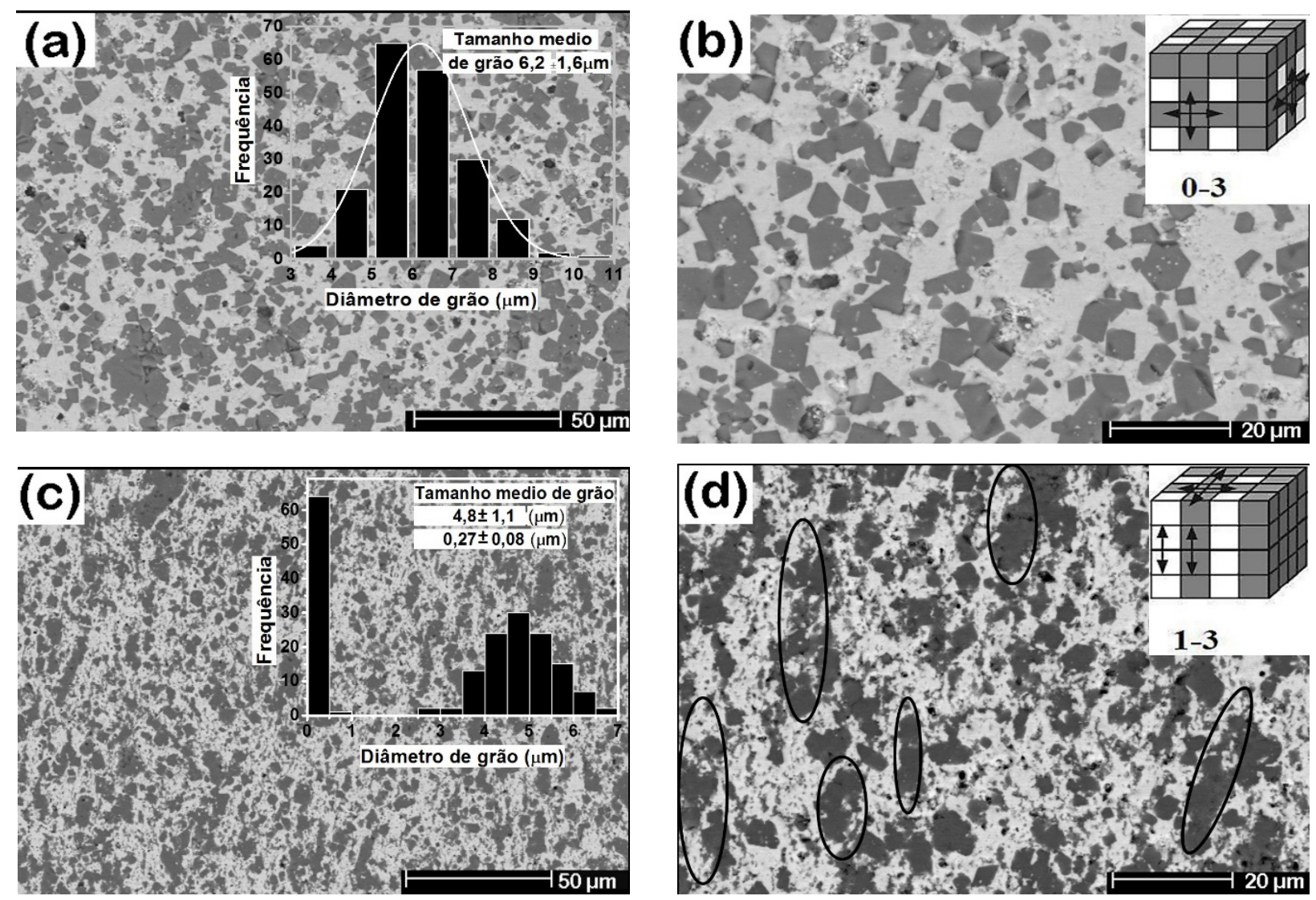

Figura 7: Micrografias obtidas por microscopia eletrônica de varredura da superfície polida do compósito PZT/FCO (50/50) sinterizado $(\mathrm{a}, \mathrm{b})$ em forno convencional e (c-d) por micro-ondas a $1125^{\circ} \mathrm{C}$ (região clara: $\mathrm{PZT}$, região escura: $\mathrm{FCO}$ ); inset $(\mathrm{a}, \mathrm{c})$ : distribuição de tamanho de grão da fase FCO.

[Figure 7: SEM micrographs of the polished surface of PZT/FCO (50/50) composite sintered (a,b) in a conventional furnace, and $(c, d)$ by microwave at $1125^{\circ} \mathrm{C}$ (bright grains: PZT, dark grains: FCO); inset (a,c): grain size distribution of FCO phase.]

na composição de maior conteúdo de ferrita (50/50). Observou-se uma distribuição estreita de tamanho de grão, tal como apresentado nos gráficos de distribuição inseridos nas micrografias de menor aumento, resultando em tamanhos médios de grão da fase ferrita de $4,5 \pm 1,1 \mu \mathrm{m}$ para

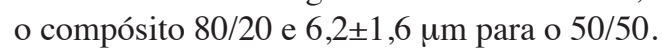

Diferentemente, nas amostras sinterizadas por microondas, as quais apresentaram distribuição bimodal de tamanho médio de grão da fase ferrita, com grãos de tamanho médio de 4,1 $\pm 1,1 \mu \mathrm{m}$ rodeados de grãos menores de $0,28 \pm 0,15 \mu \mathrm{m}$ na composição 80/20 (Fig. 6c) e valores de tamanho médio de grãos de $4,8 \pm 1,1 \mu \mathrm{m}$ e $0,27 \pm 0,08 \mu \mathrm{m}$ para a composição 50/50 (Fig. 7c), observou-se que principalmente os grãos menores apresentaram certa direção preferencial de distribuição, tal como ressaltado nas elipses marcadas nas micrografias de maior aumento (Figs. 6d e 7d), distribuição que pode ser relacionada a um esquema de conectividade local tipo 1-3 e 2-3, conforme modelo do cubo inserido nas Figs. 6d e 7d. Na análise geral, entretanto, concluiuse que a conectividade geral associada à amostra foi $0-3$, resultado similar aos reportados por Sheikh et al. $[4,6]$, que verificaram, nos compósitos magnetoelétricos bifásicos, vários esquemas de conectividade local na amostra, resultado associado à complexidade do comportamento de percolação de nanopartículas distribuídas em uma matriz também nanométrica [6-11]. Por outro lado, a tendência preferencial de distribuição da fase minoritária na matriz de PZT, nas amostras sinterizadas por micro-ondas, pode estar também associada com a intensificação dos mecanismos de difusão e mecanismos não térmicos que ocorrem apenas na sinterização assistida por micro-ondas [15], que favorecem o grau de percolação da fase ferrita em certa direção preferencial, provavelmente na direção de um máximo do campo eletromagnético, sendo mais acentuado esse efeito em materiais de partida nanométricos.

A caracterização dielétrica, resistividade elétrica à temperatura ambiente e coeficiente magnetoelétrico foram determinados nas amostras sinterizadas tanto por microondas como convencional. A composição 80/20 apresentou valores de resistividade da ordem de $10^{11}$ e $10^{10} \Omega . m$ correspondentes aos compósitos sinterizados por microondas e por forno convencional, respectivamente. Nos compósitos 50/50, a resistividade foi de $10^{7}$ e $10^{6} \Omega . \mathrm{m}$ nos sinterizados por micro-ondas e forno convencional, respectivamente; a redução esperada foi decorrente do aumento da fase ferrita em relação ao compósito anterior. 
Tabela II - Densidade relativa, tamanho médio de grãos, resistividade elétrica, constante dielétrica ( $\left.\varepsilon^{\prime}\right)$, temperatura de Curie $(\mathrm{Tc})$, campo magnético $\left(\mathrm{H}_{\max }\right)$ e coeficiente magnetoelétrico máximo $\left(\alpha_{33}\right)$ dos compósitos sinterizados a $1125^{\circ} \mathrm{C}$.

[Table II - Relative density, average grain size, electrical resistivity, dielectric constant $\left(\varepsilon^{\prime}\right)$, magnetic field $\left(H_{\max }\right)$, and maximum magnetoelectric coefficient $\left(\alpha_{33}\right)$ of the sintered composites at $1125^{\circ} \mathrm{C}$.]

\begin{tabular}{ccccc}
\hline & \multicolumn{2}{c}{ SC } & \multicolumn{2}{c}{ SM } \\
& $80 / 20$ & $50 / 50$ & $80 / 20$ & $50 / 50$ \\
\hline Densidade relativa $(\%)$ & 93,5 & 97,4 & 90,5 & 92,0 \\
Tamanho médio de grãos FCO & $4,54 \pm 1,13$ & $6,2 \pm 1,6$ & $4,05 \pm 1,10$ & $4,8 \pm 1,1$ \\
$(\mu \mathrm{m})$ & $1,5 \times 10^{10}$ & $5,7 \times 10^{6}$ & $2,0 \times 10^{11}$ & $7,5 \times 10^{7}$ \\
Resistividade elétrica $(\Omega . m)$ & 22925 & 8843 & 6877 & 5129 \\
$\varepsilon_{\text {max }}^{\prime}(1 \mathrm{kHz})$ & 354 & 380 & 350 & 359 \\
$\mathrm{Tc}\left({ }^{\circ} \mathrm{C}\right)$ & 6050 & 3224 & 3410 & 1878 \\
$\varepsilon_{\text {max }}^{\prime}(1 \mathrm{MHz})$ & 365 & 396 & 412 & 478 \\
$\mathrm{Tc}\left({ }^{\circ} \mathrm{C}\right)$ & 1,9 & 3,0 & 1,4 & 3,5 \\
$\mathrm{H}_{\max }(\mathrm{kOe})$ & 1,44 & 2,81 & 0,14 & 0,94 \\
$\alpha_{33 \mathrm{ME}}$ & &
\end{tabular}

De acordo com a Tabela II, os valores de resistividade das amostras sinterizadas por micro-ondas foram de ordem superior aos dos compósitos sinterizados convencionalmente, com densidades relativas pouco maiores do que as amostras sinterizadas por micro-ondas. Esses valores de densidade, um pouco inferiores nos compósitos sinterizados por microondas, podem estar atrelados à pequena volatilização de $\mathrm{PbO}$ do PZT, conforme discutido anteriormente e observado por outros autores [20], já que se sabe que a célula unitária que contém $\mathrm{PbZrO}_{3}$ (da solução sólida $\mathrm{PbZrO}_{3}-\mathrm{PbTiO}_{3}$ ) tem estabilidade química menor que a de $\mathrm{PbTiO}_{3}$ [20]. Assim, quando ocorre uma pequena perda de $\mathrm{PbO}$, a densidade aparente é um pouco inferior à da densidade teórica do PZT, inferindo-se que os valores de densidades relativas calculados para os compósitos sinterizados por micro-ondas, levandose em consideração as densidades teóricas dos compósitos com $50 \%$ e $20 \%$ de fase ferrita $\left(6,66 \mathrm{~g} / \mathrm{cm}^{3}\right.$ e $7,47 \mathrm{~g} / \mathrm{cm}^{3}$, respectivamente), podem estar um pouco acima da densidade aparente do material. Além disso, a análise dos resultados da resistividade elétrica é uma ferramenta para a verificação de difusão e, consequentemente, do grau de integridade entre as fases constituintes dos compósitos [16,21]. Tendo em conta que a resistividade do PZT foi da ordem de $10^{12} \Omega . \mathrm{m}$ e da FCO de $10^{6} \Omega . m$, espera-se um aumento da condutividade elétrica da fase ferroelétrica, quando contaminada com íons de ferro, ou do compósito, pela percolação de possíveis fases espúrias (condutivas) formadas pela difusão entre as duas fases [16, 21]. Assim, os altos valores de resistividade obtidos neste trabalho reiteram que a integridade das fases foi conservada nos dois métodos de sinterização, sendo mais eficiente na sinterização por micro-ondas.

A variação da constante dielétrica $\left(\varepsilon^{\prime}\right)$ em função da temperatura dos compósitos 80/20 e 50/50 nas frequências de $1,10,100$ e $1000 \mathrm{kHz}$ está apresentada na Fig. 8. Observou-se que todas as constantes dielétricas das amostras aumentaram com o aumento da temperatura e apresentaram um pico em uma temperatura em particular, denominada temperatura de Curie (Tc), a qual é característica do comportamento ferroelétrico. Observou-se que, com o aumento da fração da fase ferrita, o pico da constante dielétrica se tornou mais largo e os valores máximos de $\varepsilon$ ' diminuíram, além de aumentar a temperatura de transição (Tabela II). Dessa forma, as curvas representaram uma transição de fase tipo difusa em todas as amostras. Considerando-se o comportamento ferroelétrico normal da fase PZT pura [22], os compósitos de PZT e FCO exibiram dispersão da constante dielétrica com a frequência, devido ao aumento da polarização interfacial com o aumento de fase FCO. A incorporação de fase não ferroelétrica (FCO) na fase ferroelétrica pura (PZT) causou um efeito de diluição das propriedades ferroelétricas nos compósitos, resultando na redução da constante dielétrica e no alargamento do pico. Esse efeito também foi associado com as heterogeneidades microscópicas entre as duas fases, tais como poros e discrepâncias entre os grãos de cada uma das fases.

As curvas da variação do coeficiente magnetoelétrico (ME), $\alpha_{33}$, das duas composições sinterizadas, tanto por micro-ondas como por forno convencional, em função do campo magnético dc e eletricamente polarizadas $(2 \mathrm{kV} / \mathrm{cm}$, durante 30 min à temperatura ambiente), estão apresentadas na Fig. 9. Observou-se que, para todos os compósitos, o valor do coeficiente ME teve um comportamento crescente passando por um valor máximo, em um determinado campo magnético denominado $\mathrm{H}_{\max }$; na Tabela II são apresentados os valores de ME máximo e $\mathrm{H}_{\max }$. Verificou-se que os compósitos sinterizados por micro-ondas apresentaram menor valor de coeficiente ME máximo, efeito possivelmente associado à pequena presença de poros e fases secundárias, que podem distorcer a conectividade local, causando uma desmagnetização e despolarização local, tal como reportado em [6]. De acordo com [23], é de se esperar que este tipo de 

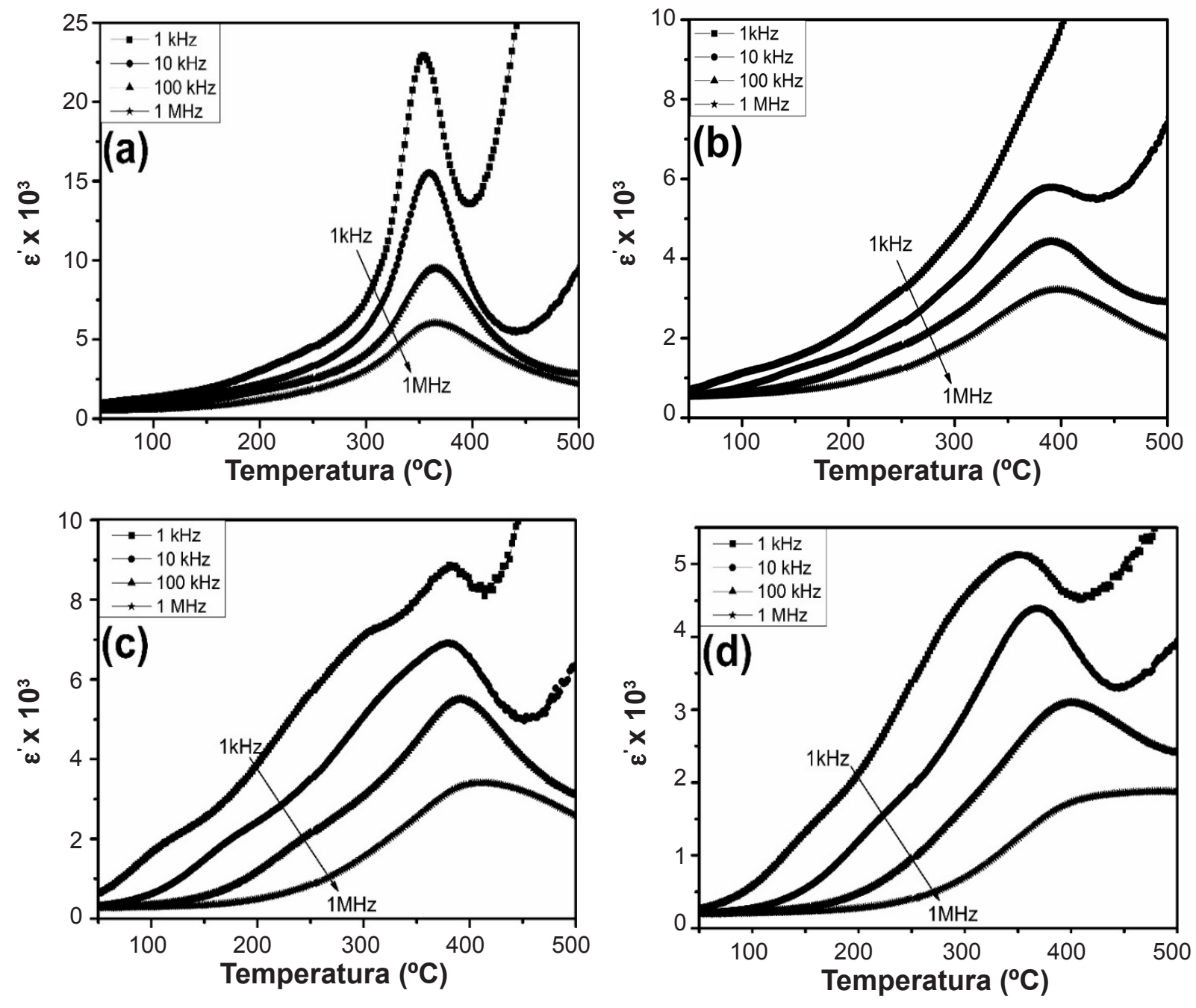

Figura 8: Constante dielétrica em função da temperatura do compósito (PZT/FCO) 80/20 sinterizado (a) convencionalmente e (b) por micro-ondas, e do compósito 50/50 sinterizado (c) convencionalmente e (d) por micro-ondas.

[Figure 8: Dielectric constant as a function of temperature of the composite (PZT/FCO) 80/20 sintered by (a) conventional route and (b) microwave, and of the composite 50/50 sintered by (c) conventional route and (d) microwave.]

defeitos (poros e fases secundárias) na estrutura tenha influência na interação do acoplamento magnetoelétrico por meio de dois mecanismos: A) o aumento de defeitos causa uma diminuição nos coeficientes piezoelétrico e piezomagnético efetivos, e B) os defeitos na estrutura amortecem as vibrações elásticas e aumentam a perda de energia gerando uma diminuição global do coeficiente ME. Outro fator a ser considerado de a resposta magnetoeléctrica ter sido menor nas amostras sinterizadas por micro-ondas é o tamanho de grão ser bem inferior em comparação com os obtidos por sinterização convencional. Alguns trabalhos, como [24, 25], demostraram que os valores do coeficiente magnetoelétrico aumentam com o aumento do tamanho médio de grãos, como resultado da melhoria da estrutura de domínio nos compósitos.

Comparando-se cada uma das composições e o tipo de sinterização utilizada, observou-se que os valores de $\alpha_{33}$ aumentaram com o aumento da fase ferrita na composição $50 / 50$, tal como foi predito em $[8,10]$, onde o máximo valor de coeficiente ME é atingido por compósitos com concentrações próximas a 50\% de cada uma das fases. No entanto, segundo resultados experimentais reportados na literatura, com o aumento da concentração da fase ferrita tem-se uma diminuição considerável da resposta ME devido ao aumento na condutividade elétrica [8-11]. Assim, os resultados do coeficiente magnetoelétrico apresentados neste trabalho confirmaram a integridade das fases constituintes PZT e FCO. O deslocamento do valor do campo $\mathrm{H}_{\max }$ para menores ou maiores valores é dependente da concentração de fase ferrita; assim, para a composição 80/20, o valor de $\mathrm{H}_{\max }$ foi de 1,4 e 1,9 kOe nas amostras sinterizadas por microondas e convencionalmente, respectivamente, enquanto para a composição 50/50 os valores de $\mathrm{H}_{\max }$ foram de 3,5 e 3,0 kOe, respectivamente, conforme apresentados na Tabela II, resultado também associado ao fato de a magnetização de saturação dos compósitos aumentar linearmente com o incremento da fase ferrimagnética, resultando, então, na diminuição dos parâmetros magnéticos com o aumento de concentração da fase não magnética (PZT) no compósito [26, 27]. 


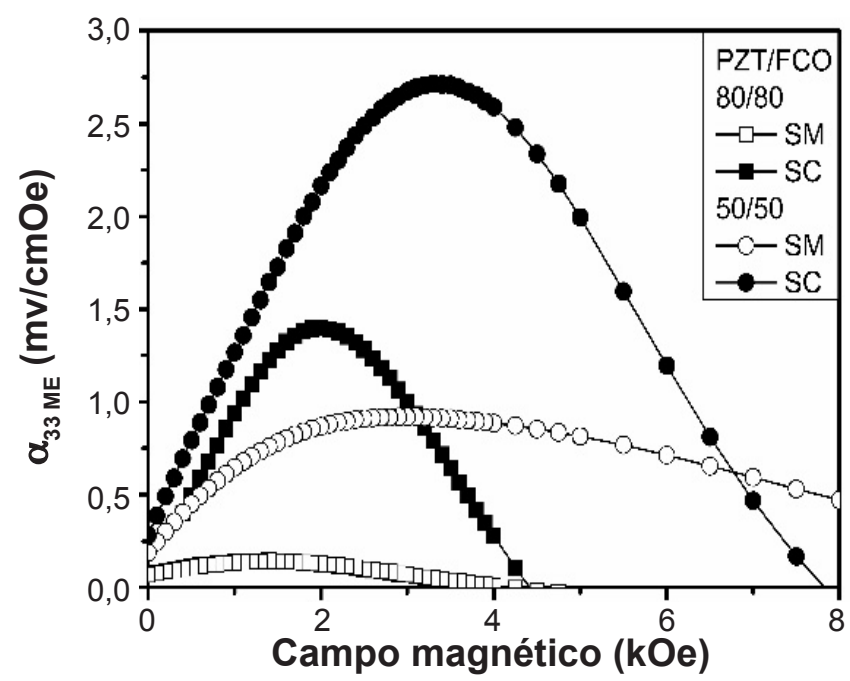

Figura 9: Coeficiente ME em função do campo magnético dos compósitos $\mathrm{PZT} / \mathrm{FCO}$ sinterizados por micro-ondas (SM) e convencionalmente (SC).

[Figure 9: ME coefficient as a function of magnetic field of PZT/FCO composites sintered by the microwave (MS) and conventional (CS) routes.]

\section{CONCLUSÕES}

O método alternativo de mistura por ultrassom foi altamente eficiente na redução do tempo de mistura, desaglomeração com ausência de contaminação e excelente distribuição granulométrica da fase ferrita na matriz de PZT, preservando-se após a sinterização tanto por microondas como convencional. Os compósitos particulados sinterizados tanto convencionalmente como por micro-ondas apresentaram microestruturas com conectividades globais 0-3, mas os compósitos submetidos à sinterização ultrarrápida assistida por micro-ondas apresentaram conectividades locais 1-3, resultado atribuído aos processos de difusão diferenciados que ocorreram na sinterização assistida por micro-ondas. Pelos altos valores de resistividade obtidos, comprovou-se que a integridade das fases foi conservada nos dois métodos de sinterização, sendo mais eficiente na sinterização por micro-ondas. $\mathrm{O}$ pico da constante dielétrica em todas as amostras foi largo e decrescente, comportamento atribuído ao fato de regiões ferroelétricas estarem rodeadas por regiões não ferroelétricas, tornando o comportamento da constante dielétrica nos compósitos do tipo relaxor, efeito evidente com o aumento da concentração de ferrita. A diminuição dos valores de coeficiente magnetoelétrico apresentada pelos compósitos sinterizados por micro-ondas, em comparação aos sinterizados convencionalmente, foi diretamente relacionada com a redução do tamanho médio de grão obtido com esse tipo de sinterização.

\section{AGRADECIMENTOS}

Os autores agradecem à Fundação de Amparo à Pesquisa do Estado de São Paulo (FAPESP) pelo apoio financeiro
(Processo n. 2008/04025-0), ao Conselho Nacional de Desenvolvimento Científico e Tecnológico (CNPq) pela bolsa concedida e ao programa de Pós-graduação em Ciência e Engenharia de Materiais da Universidade Federal de São Carlos.

\section{REFERÊNCIAS}

[1] M. Fiebig, J. Phys. D: Appl. Phys. 38, 8 (2005) R123.

[2] M.A. Rahman, M.A. Gafur, A.K.M. Akther Hossain, J. Magn. Magn. Mater. 345, 1 (2013) 89.

[3] S. Roy, S.B. Majumder, J. Alloys Compd. 538, 1 (2012) 153.

[4] A.D. Sheikh, V.L. Mathe, Mater. Chem. Phys. 119, 3 (2010) 395.

[5] W. Eerenstein, N.D. Mathur, J.F. Scott, Nature 442, 1 (2006) 759 .

[6] A.D. Sheikh, V.L. Mathe, Smart. Mater. Struct. 18, 6 (2009) 65014.

[7] N.A. Spaldin, M. Fiebig, Sci. 309, 5733 (2005) 391.

[8] C.W. Nan, M.I. Bichurin, S. Dong, D. Viehland, G. Srinivasan, J. Appl. Phys. 103, 3 (2008) 31101.

[9] G. Lawes, G. Srinivasan, J. Phys. D: Appl. Phys. 44, 24 (2011) 243001.

[10] C.W. Nan, Phys. Rev. B. 50, 9 (1994) 6082.

[11] R. Rani, J.K. Juneja, S. Singh, C. Prakash, K.K. Raina, J. Magn. Magn. Mater. 345, 1 (2013) 55.

[12] R.F.K. Gunnewiek, R.H.G.A. Kiminami, Ceram. Int. 40, 1 (2014) 10667.

[13] P.M. Souto, M.A. Camerucci, A.G. Tomba-Martinez, R.H.G.A. Kiminami. J. Eur. Ceram. Soc. 31, 15 (2011) 2819. [14] P.M. Souto, R.R. Menezes, R.H.G.A. Kiminami, Ceram. Int. 37, 1 (2011) 241.

[15] R.R. Menezes, P.M. Souto, R.H.G.A. Kiminami, in Sintering of ceramics - new emerging techniques, A. Lakshmanan (Ed.), InTech (2012) 3.

[16] F.L. Zabotto, A.J. Gualdi, A.J.A. de Oliveira, J.A. Eiras, D. Garcia, Ferroelectr. 428, 1 (2012) 122.

[17] J. Ryu, A. Vasquez-Carazo, K. Uchino, H.E. Kim. J. Electroceram. 7, 1 (2001) 17.

[18] L. Iyengar, B.R. Prasad, B. Quadri. Curr. Sci. 42, 15 (1973) 534.

[19] S.N. Kallaev, G.G. Gadjiev, I.K. Kamilov, S.A. Sadykov, Z.M. Marov, R.M. Ferzilaev, Itegr. Ferroelectr. 72, 1 (2006) 23.

[20] G. Shirani, F. Jona, Ferroelectric crystals, Dover Public. (1993).

[21] C.M. Kanamadi, B.K. Das, C.W. Kim, D.I. Kang, H.G. Cha, E.S. Ji, A.P. Jadhav, B.E. Jun, J.H. Jeong, B.C. Choi, B. K. Chougule, Y.S. Kang, Mater. Chem. Phys. 116, 1 (2009) 6.

[22] V.M. Petrov, G. Srinivasan, U. Laletsin, M.I. Bichurin, D.S. Tuskov, N. Paddubnaya, Phys. Rev. B: Condens. Matter. 75, 17 (2007) 174422.

[23] R. Yimnirun, S. Ananta, P.L. Songklanakarin, J. Sci. Technol. 26, 4 (2004) 529.

[24] R.A. Islam, S. Priya, J. Mater. Sci. 43, 10 (2008) 3560. 
[25] F.L. Zabotto, A.J. Gualdi, P.C. de Camargo, A.J.A. de Oliveira, J.A. Eiras, D. Garcia, J. Alloys Compd. 676, 1 (2016) 80.

[26] M.A. Ahmeda, S.F. Mansour, M. Afifi, J. Alloys Compd.
578, 1 (2013) 303.

[27] R.C. Kambale, K.M. Song, N. Hur, Curr. Appl. Phys. 13, 3 (2013) 562.

(Rec. 15/06/2016, Rev. 29/09/2016, 28/12/2016, Ac. $28 / 12 / 2016)$ 